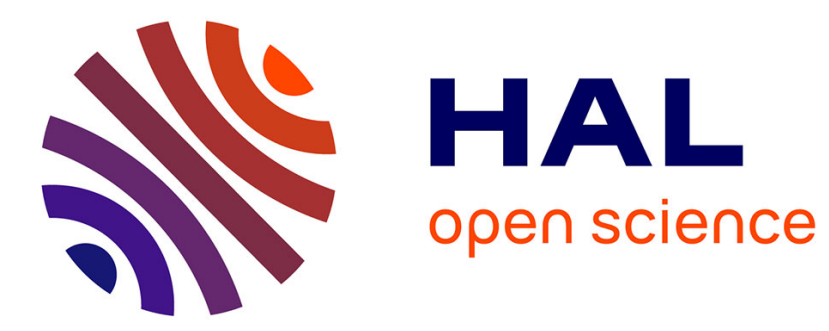

\title{
Using Sparse Array for 3D Passive Cavitation Imaging
} Audrey Sivadon, François Varray, Barbara Nicolas, Jean-Christophe Béra, Bruno Gilles

\section{To cite this version:}

Audrey Sivadon, François Varray, Barbara Nicolas, Jean-Christophe Béra, Bruno Gilles. Using Sparse Array for 3D Passive Cavitation Imaging. 2020 IEEE International Ultrasonics Symposium (IUS), Sep 2020, Las Vegas, United States. pp.1-4, 10.1109/IUS46767.2020.9251562 . hal-03070225

\section{HAL Id: hal-03070225 \\ https://hal.science/hal-03070225}

Submitted on 4 Jan 2021

HAL is a multi-disciplinary open access archive for the deposit and dissemination of scientific research documents, whether they are published or not. The documents may come from teaching and research institutions in France or abroad, or from public or private research centers.
L'archive ouverte pluridisciplinaire HAL, est destinée au dépôt et à la diffusion de documents scientifiques de niveau recherche, publiés ou non, émanant des établissements d'enseignement et de recherche français ou étrangers, des laboratoires publics ou privés. 


\section{Using Sparse Array for 3D Passive Cavitation Imaging}

\author{
Audrey Sivadon \\ Université Lyon 1, Inserm, U1032 \\ LabTau \\ Lyon, France \\ audrey.sivadon@inserm.fr \\ Jean-Christophe Béra \\ Université Lyon 1, Inserm, U1032 \\ LabTau \\ Lyon, France \\ jean-christophe.bera@inserm.fr
}

\author{
François Varray \\ INSA, University of Lyon, CNRS \\ CREATIS \\ Lyon, France \\ francois.varray@creatis.insa-lyon.fr \\ Bruno Gilles \\ Université Lyon 1, Inserm, U1032 \\ LabTau \\ Lyon, France \\ bruno.gilles@inserm.fr
}

\author{
Barbara Nicolas \\ INSA, University of Lyon, CNRS \\ CREATIS \\ Lyon, France \\ barbara.nicolas@creatis.insa-lyon.fr
}

Index Terms-Passive Acoustic Mapping, 3D Imaging, Cavitation Imaging, Sparse Array

\section{INTRODUCTION}

Cavitation is involved in a number of therapeutic applications of ultrasound (US) [1]-[3]. Monitoring these treatments requires real-time imaging of the cavitation activity during treatment. For this reason, Passive Acoustic Mapping (PAM) of cavitation has drawn an increasing attention during the past years [4]-[8].

There is a need to extend the beamforming algorithms of PAM to volumetrix imaging and this work has been recently enhanced by the scientific community - first with a 3D ultrasound tomography system [9] and second with a 1024element transducer and singular value decomposition [10] for pulsed cavitation US therapy in moving tissues. However, such systems are very complex because it imposes the synchronisation of four Vantage systems, the separation of the signal on each sub-part on the various systems, and the difficulty to obtain real-time imaging based on the full probe [11]. For these reasons, the interest to reduce the number of connected elements while keeping the same contrast and resolution is of major interest as already proposed for active imaging with optimized or not strategies [12]-[14].

In this work, we propose a method for 3D real-time frequency domain Delay-And-Sum (DAS) using a multiplex transmitter of 1024 elements that can be connected to a single Verasonics system. However, compared to a commonly used linear array with often 128 sensors, the use of the matrix array implies costly acquisition system and an increased time computation making the real-time application less affordable. With the proposed solution, the signals are directly acquired, processed and displayed on a single system which affords to envisage more easily a clinical transfer. The objective in this paper is then to study 3 sparse probes with different arrangements and investigate if whether or not the results obtained in active imaging with this type of probes are reproducible with passive imaging.

\section{METHOD}

\section{A. Simulated acquisition probes and sources}

The probe considered in this paper is a Vermon $3-\mathrm{MHz}$ matrix probe composed of 1024 sensors with 32 elements along the $x$-axis and 35 elements, including 3 dead lines, along the $y$-axis as shown in Fig. 1 (a). The spacing between each element (center to center distance) is $0.3 \mathrm{~mm}$ in each direction. The use of a Verasonics Universal Transducer Adapter (UTA) with MUX switch topology allows almost arbitrary sparse configurations of the 1024-sensor probe.

With taking into account the technical constraints of the probe, 3 sparse configurations have been simulated for study with 2 regular patterns and 1 random (Fig. 1 (b,c,d)). The interest of the random configuration is the absence of regularity in the position of the active elements, which aims at suppressing grating lobes for the reconstruction.

We simulate a $4 \mathrm{MHz}$ harmonic point source centered with the probe along the $x$ and $y$ axis and at an axial distance of $20 \mathrm{~mm}$. The time duration of the simulation is $600 \mu$ s sampled at a frequency of $20 \mathrm{MHz}$. To be more representative of an experimental acquisition, we simulate an additive white Gaussien acquisition noise, with a Signal-toNoise Ratio (SNR) of $10 \mathrm{~dB}$ at the frequency of interest.

\section{B. 3D Frequency-Domain Passive-Delay-And-Sum (3D FD- PDAS) algorithm}

a) $2 D$ PDAS with the CSM formulation: In this paper, we use a Delay-And-Sum (DAS) method in the frequency domain using an estimation of the Cross Spectral Matrix (CSM) [15], [16].

Considering a linear array of $N$ receiving elements, the matrix containing the time samples of each element and 

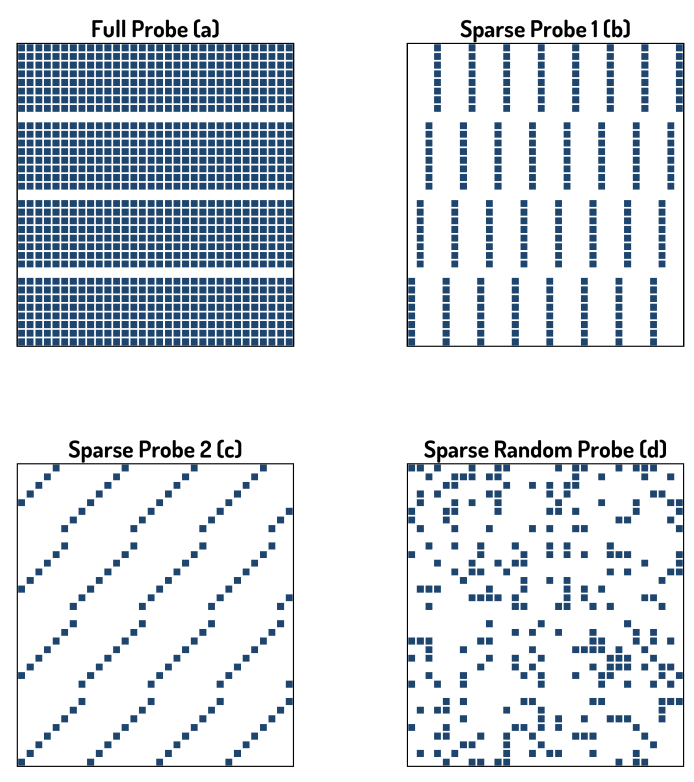

Fig. 1. Full (a) and sparse (b,c,d) configurations for the 1024-channel multiplexed probe. The blue squares represent the active elements.

its Fourier transform are respectively $\mathbf{s}(t)$ and $\mathbf{S}(f)$. The corresponding $(N \times N) \mathrm{CSM}$ is

$$
\mathbf{M}(f)=E\left\langle\lim _{T \rightarrow+\infty} \frac{1}{T} \mathbf{S}(f) \mathbf{S}^{*}(f)\right\rangle,
$$

where $T$ is the recording time, $E\langle\rangle,()^{*}$ the expectation and conjugate transpose. The estimation of the CSM consists of partitioning the temporal data into $K$ artificial realizations of duration $T_{\text {snap }}$, computing each corresponding CSM by mean of its Fourier transform, and averaging on the number of realizations $K$ as

$$
\hat{\mathbf{M}}(f)=\frac{1}{T_{\text {snap }}} \frac{1}{K} \sum_{k=1}^{K} \mathbf{S}_{k}(f) \mathbf{S}_{k}^{*}(f) .
$$

The formulation in the frequency domain of DAS algorithm for Passive Acoustic Mapping is then

$$
\hat{P}(\overrightarrow{\mathbf{r}}, f)=\mathbf{h}^{*}(\overrightarrow{\mathbf{r}}, f) \hat{\mathbf{M}}(f) \mathbf{h}(\overrightarrow{\mathbf{r}}, f) .
$$

with $\mathbf{h}(\overrightarrow{\mathbf{r}}, f)$ and $\hat{P}(\overrightarrow{\mathbf{r}}, f)$ respectively the steering vector and the estimated power of the source as a function of the space $\overrightarrow{\mathbf{r}}$ and the frequency $f$.

b) From $2 D$ FD-PDAS to $3 D$ FD-PDAS: The dimensions of the simulated data $\mathbf{y}(t)$ with a 1D probe is $\left(N_{t} \times N\right)$ with $N_{t}$ the number of time samples and $N$ the number of elements in the linear array. While the natural way of ordering the simulated data with a $2 \mathrm{D}$ probe would be to separate the $x$ and $y$ dimensions, thus adding in all the computations one dimension, we choose to keep the same formulation with an $\left(N_{t} \times N\right)$ matrix $\hat{M}$ with $N$ the total number of elements in the

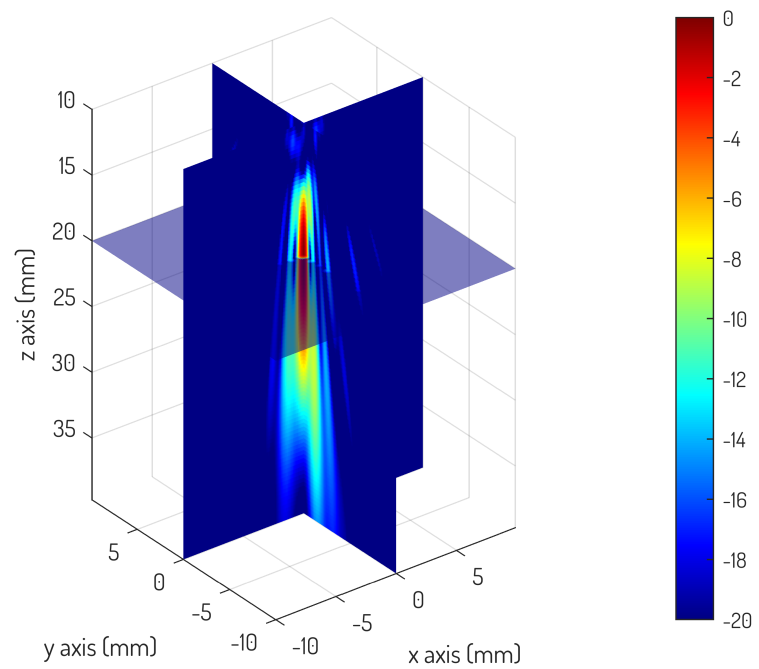

Fig. 2. Three perpendicular slices of the 3D passive cavitation map of a $4 \mathrm{MHz}$ harmonic simulated source at $[0,0,20] \mathrm{mm}$, reconstructed at the frequency $4 \mathrm{MHz}$ using the 3D-FD-DAS algorithm, with $0.1 \mathrm{~mm}$ resolution for the $x$ and $y$ axis and $0.3 \mathrm{~mm}$ resolution for the $z$ axis.

matrix array. This way, the formulation of the 3D FD-PDAS in equation (3) can be adapted from the 2D case by simply adding a dimension to the positions of the chosen reconstructed area, as well as to the positions of the elements of the probe.

\section{RESUlts AND Discussion}

a) Reconstruction of a $3 D$ passive cavitation map: On Fig. 2, three slices of a reconstructed volume are shown. The CSM estimation is done with $K=300$ snapshots and an overlap of $90 \%$ for the partitioning which results in a time duration of $T_{\text {snap }}=20 \mu$ s, i.e. 80 periods of a $4 \mathrm{MHz}$ sinusoidal wave in a snapshot. The resolution of the reconstructed volume is $0.1 \mathrm{~mm}$ for the $x$ and $y$ axes and $0.3 \mathrm{~mm}$ for the $z$ axis. The resulting image is very similar to typical results obtained with a 2D FD-PDAS algorithm.

b) Probe's configuration comparison: On Fig. 3, the $z=20 \mathrm{~mm}$ c-scan is shown for the 4 different probes. Concerning the full probe, on Fig. 3 (a), the c-scan shows a primary lobe centered on the source position $[0,0,20] \mathrm{mm}$ and secondary lobes along the $x$ and $y$ axes. The absence of symmetry between the $x$ and $y$ axes in the pattern of the secondary lobes is a consequence of the presence of the 3 dead lines represented on Fig. 1 (a). The probe is thus slightly longer on the $y$ axis direction.

The two regular configurations on Fig. 3 (b,c) present as expected grating lobes that can alter the performance of the source localization mostly in the presence of more than one source in the volume. The angle of the grating lobes orientations and the distance between each grating lobe depend directly on the direction and spacing of the regular pattern of the probe. Interestingly, the sparse random probe, Fig. 3 (d), does not exhibit grating lobes, due to the disordered nature 

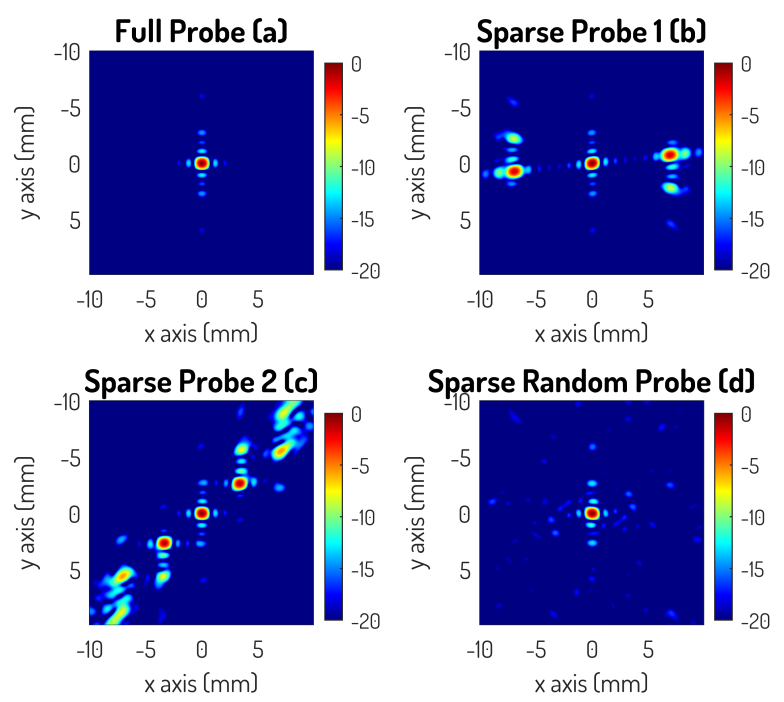

Fig. 3. C-scans at $z=20 \mathrm{~mm}$ of the reconstructed 3D cavitation mapping of a $4 \mathrm{MHz}$ harmonic simulated source at $[0,0,20] \mathrm{mm}$, reconstructed at the frequency $4 \mathrm{MHz}$ using the 3D-FD-DAS algorithm, with $0.1 \mathrm{~mm}$ resolution for both axis. (a) represents the use of the full probe, (b,c,d) respectively the use of the 3 sparse probes corresponding to the ones of Fig. 1. The dynamic range is $20 \mathrm{~dB}$.

of the random pattern of the configuration. This aspect can be perceived on the irregularity of the minor lobe shapes and spatial distribution on the map. The similarity between the maps obtained with the full probe and with the sparse random configuration is shown on Fig. 4 where the projection of the maximum values along the $x$ axis of the maps of Fig. 3 is plotted. We can see the difference of amplitude between the primary lobe representing the position of the source and the grating lobes of the sparse regular configurations, at $-1 \mathrm{~dB}$ for sparse probe 1 et $-1.7 \mathrm{~dB}$ for sparse probe 2 , confirming their irrelevance in therapeutic monitoring. Besides, the projection of the sparse random probe is overlaying the full probe's curve between $\pm 2 \mathrm{~mm}$. This plot shows also that having a random sparse configuration of the probe resutls in an overall decrease of the dynamic $(+10 \mathrm{~dB}$ on average beyond the first 2 secondary lobes compared to the full probe). Finally, the reduction of the number of active elements allows to divide by almost a factor 10 the calculation duration when compared to the full probe configuration mainly because of the CSM estimation.

\section{CONCLUSION}

The use of sparse probe configurations, already studied in 3D Active Imaging, has been introduced in this paper for Passive Acoustic Mapping. Indeed, such sparse probe configurations appear to be relevant and offer the possibility of a 3D passive cavitation imaging with affordable equipment and processing time. Even if the regular patterns showed to be unusable because of the grating lobes close from the source, the random pattern seems to be a suitable candidate to combine reduction of the number of elements, from 1024 to 256 in the

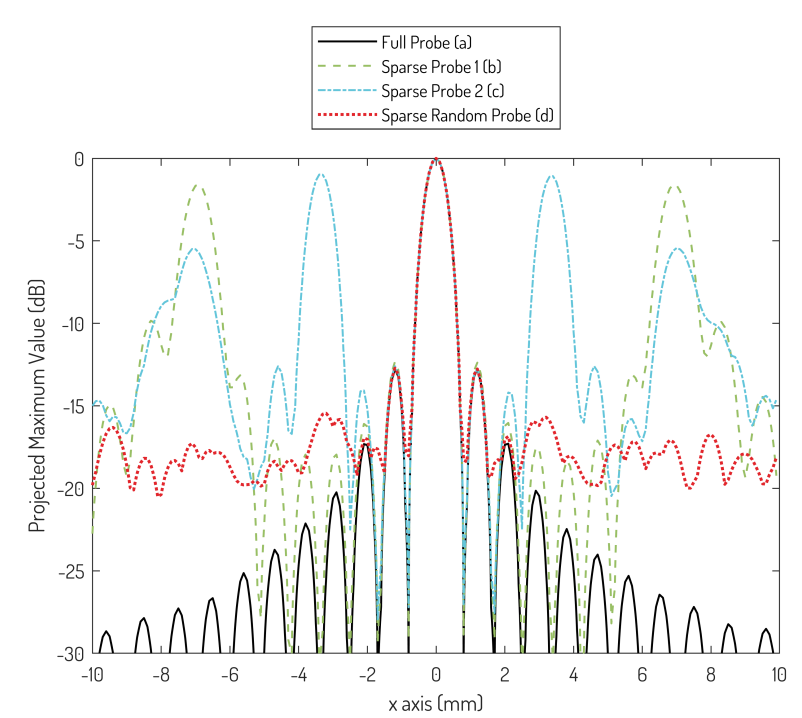

Fig. 4. Projection of the maximum value along the $x$ axis of the $z$ slices of Fig. 3.

present study, with a limited loss of image quality, resulting in an equivalent of the full probe reconstruction. Even if further investigations are needed for the comparison of the full and random sparse probes with different types of sources, and for an experimental validation of the results, its use should be of great interest for the PAM community.

\section{REFERENCES}

[1] C. C. Coussios and R. A. Roy, "Applications of acoustics and cavitation to noninvasive therapy and drug delivery," Annu. Rev. Fluid Mech., vol. 40, pp. 395-420, 2008.

[2] V. A. Khokhlova, J. B. Fowlkes, W. W. Roberts, G. R. Schade, Z. Xu, T. D. Khokhlova, T. L. Hall, A. D. Maxwell, Y.-N. Wang, and C. A. Cain, "Histotripsy methods in mechanical disintegration of tissue: Towards clinical applications," International journal of hyperthermia, vol. 31, no. 2, pp. 145-162, 2015.

[3] Y. Yang, Q. Li, X. Guo, J. Tu, and D. Zhang, "Mechanisms underlying sonoporation: Interaction between microbubbles and cells," Ultrasonics Sonochemistry, p. 105096, 2020.

[4] M. Gyöngy and C.-C. Coussios, "Passive spatial mapping of inertial cavitation during hifu exposure," IEEE Transactions on Biomedical Engineering, vol. 57, no. 1, pp. 48-56, 2009.

[5] C. Coviello, R. Kozick, J. Choi, M. Gyöngy, C. Jensen, P. P. Smith, and C.-C. Coussios, "Passive acoustic mapping utilizing optimal beamforming in ultrasound therapy monitoring," The Journal of the Acoustical Society of America, vol. 137, no. 5, pp. 2573-2585, 2015.

[6] P. Boulos, F. Varray, A. Poizat, A. Ramalli, B. Gilles, J.-C. Bera, and C. Cachard, "Weighting the passive acoustic mapping technique with the phase coherence factor for passive ultrasound imaging of ultrasoundinduced cavitation," IEEE Transactions on Ultrasonics, Ferroelectrics, and Frequency Control, vol. 65, no. 12, pp. 2301-2310, 2018.

[7] V. A. Salgaonkar, S. Datta, C. K. Holland, and T. D. Mast, "Passive cavitation imaging with ultrasound arrays," The Journal of the Acoustical Society of America, vol. 126, no. 6, pp. 3071-3083, 2009.

[8] K. J. Haworth, K. B. Bader, K. T. Rich, C. K. Holland, and T. D. Mast, "Quantitative frequency-domain passive cavitation imaging," IEEE transactions on ultrasonics, ferroelectrics, and frequency control, vol. 64, no. 1, pp. 177-191, 2016.

[9] X. Cai, J. Foiret, J. Roth, Z. Gazit, G. Pelled, D. Gazit, and K. W. Ferrara, "3D monitoring and control of microbubble cavitation for gene delivery," in 2019 IEEE International Ultrasonics Symposium (IUS). IEEE, 2019, pp. $888-890$. 
[10] D. S. Escudero, G. Goudot, M. Vion, M. Tanter, and M. Pernot, "2D and 3D real-time passive cavitation imaging of pulsed cavitation ultrasound therapy in moving tissues," Physics in Medicine \& Biology, vol. 63, no. 23 , p. $235028,2018$.

[11] L. Petrusca, F. Varray, R. Souchon, A. Bernard, J.-Y. Chapelon, H. Liebgott, W. N'Djin, and M. Viallon, "Fast volumetric ultrasound B-mode and Doppler imaging with a new high-channels density platform for advanced 4D cardiac imaging/therapy," Applied Sciences, vol. 8, no. 2, p. 200, 2018.

[12] E. Roux, F. Varray, L. Petrusca, C. Cachard, P. Tortoli, and H. Liebgott, "Experimental 3-D ultrasound imaging with 2-d sparse arrays using focused and diverging waves," Scientific Reports, vol. 8.

[13] H. Yoon and T.-K. Song, "Sparse rectangular and spiral array designs for 3D medical ultrasound imaging," Sensors, vol. 20, no. 1, p. 173, 2019.

[14] A. Ramalli, S. Harput, S. Bézy, E. Boni, R. J. Eckersley, P. Tortoli, and J. D'Hooge, "High-frame-rate tri-plane echocardiography with spiral arrays: From simulation to real-time implementation," IEEE Transactions on Ultrasonics, Ferroelectrics, and Frequency Control, vol. 67, no. 1, pp. 57-69, 2020.

[15] J. Maksym, "A robust formulation of an optimum cross-spectral beamformer for line arrays," The Journal of the Acoustical Society of America, vol. 65, no. 4, pp. 971-975, 1979.

[16] D. E. Grant, J. H. Gross, and M. Zebrick Lawrence, "Cross-spectral matrix estimation effects on adaptive beamforming," The Journal of the Acoustical Society of America, vol. 98, no. 1, pp. 517-524, 1995. 